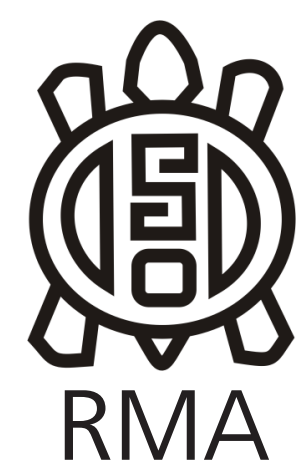

Dossier

\title{
Procesos de formación de conjuntos líticos: casos de estudio y abordajes metodológicos
}

\author{
Formation processes of lithic assemblages: case studies and \\ methodological approaches \\ *Eugenia Carranza, **Catalina Balirán, \\ ${ }^{* * *}$ Federico Bobillo $y * * * *$ Luca Sitzia
}

\begin{abstract}
*Instituto Multidisciplinario de Historia y Ciencias Humanas (IMHICIHU - CONICET). CABA, Argentina. E-mail: carranza.e89@gmail.com **Facultad de Filosofía y Letras - Universidad de Buenos Aires. CABA, Argentina. E-mail: cataclorofila@gmail.com *** Instituto Superior de Estudios Sociales (ISES-CONICET). San Miguel de Tucumán, Tucumán, Argentina. E-mail: fede_bobillo@yahoo.com.ar **** Universidad de Tarapacá, Instituto de Alta Investigación. Laboratorio de Análisis e Investigaciones Arqueométricas. Arica, Chile. E-mail: Icsitzia@gmail.com
\end{abstract}

\begin{abstract}
Resumen
El registro arqueológico lítico de superficie se encuentra expuesto a diversos agentes postdepositacionales, que desencadenan una serie de modificaciones que son parte de los procesos de formación de los conjuntos que hallamos. El estudio de estas modificaciones brinda información indispensable a la hora de proponer interpretaciones conductuales sobre los grupos humanos del pasado. En este trabajo se destaca la relevancia que tiene la aplicación de un programa tafonómico al estudio de conjuntos líticos superficiales. Para ello, se consideraron los avances realizados en tres casos de estudio en zonas desérticas/semidesérticas de Argentina. Entre las metodologías utilizadas en cada caso se encuentran observaciones naturalistas, muestreos, experimentaciones y estudios a escala del artefacto, por ejemplo, análisis de la abrasión eólica. A partir de las problemáticas analizadas en cada caso se pudo reconocer el rol de la erosión eólica y/o circulación de aguas superficiales, o el pisoteo animal, como agentes de transformación que inciden en la estabilidad de los conjuntos líticos superficiales y en sus atributos cuantitativos y cualitativos. El armado de un programa tafonómico permite analizar los efectos generados por los procesos postdepositacionales y conocer la naturaleza preservada o transformada de los contextos líticos con los cuales se trabaja.
\end{abstract}

Palabras clave: Lítico; Tafonomía; Procesos de formación; Patagonia; Antofagasta de la Sierra.

\begin{abstract}
The surface lithic archaeological record is exposed to various post-depositional agents, which trigger a series of modifications which are part of the formation processes of the assemblages that archaeologists recover. The study of these modifications provides essential information when proposing behavioral interpretations about past human groups. This paper highlights the relevance of the application of a taphonomic program to the study of surface lithic assemblages. To accomplish this, the advances made in three case studies of desert / semi-desert zones of Argentina were considered. Among the methodologies developed in each case are naturalistic observations, sampling, experimentation and studies at the artifact scale, for example, analysis of wind abrasion. From the problems analyzed in each case it was possible to recognize the role of wind erosion and / or circulation of surface water, or the trampling of fauna, which are agents of transformation that affect the stability of surface lithic assemblages and their quantitative and qualitative attributes. The assembly of a taphonomic program allows us to analyze the effects generated by post-depositional processes and to know the preserved or transformed nature of the lithic contexts with which we work.
\end{abstract}

Keywords: Lithic; Taphonomy; Formation Processes; Patagonia; Antofagasta de la Sierra.

\section{Introducción}

El estudio de las formas en las que el registro puede presentarse ha llevado al desarrollo de una serie de marcos teórico-metodológicos que se enfocan en las complejas relaciones sistémicas existentes entre factores culturales, biológicos y físicos, y los procesos de formación (Schiffer, 1972, 1983; Butzer, 1982). Dichos estudios buscan determinar cómo estos factores afectan el registro hallado por los arqueólogos en la actualidad. Específicamente, la 
tafonomía, se ocupa de los mecanismos de transformación que afectan al registro arqueológico desde el momento de su depositación hasta su eventual recuperación (Hiscock, 1985; Lyman, 1994; Borrero, 2001; Borrazzo, 2010; Domínguez-Rodrigo, Fernández-López y Alcalá, 2011). Este trabajo tiene como objetivo comparar tres casos de estudio tafonómicos realizados sobre conjuntos líticos de superficie en ambientes desérticos y semidesérticos (Figura 1). Específicamente se busca identificar las similitudes y diferencias que presenta cada uno de ellos. Asimismo, se da cuenta de la contribución de la tafonomía lítica y los procesos de formación de sitio a la problemática arqueológica que presenta cada caso.

\section{Metodología.}

Los tres casos presentados a continuación se enmarcan en el enfoque de la tafonomía lítica (Borrazzo, 2010) y procesos de formación de sitio. Una primera etapa consistió en utilizar información geoarqueológica para contextualizar los conjuntos en función del ambiente. En este paso la realización de observaciones naturalistas fue clave. Esto permitió la identificación de los agentes tafonómicos que actúan o actuaron sobre los conjuntos líticos recuperados. Luego como paso previo al análisis tecnomorfológico, se realizó el análisis tafonómico de los conjuntos. En cada uno de los casos las variables seleccionadas para el análisis derivaron de las observaciones realizadas en el primer paso. La evaluación de todas las variables, habilita a comprender las condiciones de mayor o menor estabilidad a las que se encuentran expuestas las piezas bajo estudio. Las variables analizadas fueron: Corrasión, se registró presencia, extensión e intensidad (en casos 1 y 3; Borrazzo, 2010); Meteorización química se registró presencia, extensión e intensidad (en caso 2; Hiscock, 1985; Borrazzo, 2010; Balirán, 2014); Carbonatos, se registró presencia y extensión (en caso 1; Dorn, 2009); Granulometría artefactual (Casos 1 y 3); Fábrica (Caso 3; Bertran, Lenoble, Todisco, Desrosiers y Sørensen, 2012). Finalmente, los casos 1 y 2 incluyeron un programa experimental pensado específicamente para evaluar las dinámicas locales y sus efectos sobre los conjuntos (i.e. desplazamientos, inversión de piezas, pérdida de materiales, pisoteo). En ambos casos se realizaron experimentos longitudinales a largo plazo que incluyeron el sembrado de pistas experimentales con relevamientos periódicos (i.e. anuales). En ambos casos los artefactos experimentales se realizaron sobre materias primas locales (sílices y vulcanitas básicas en el caso 1 y lutita en el caso 2). En el caso 1 las pistas experimentales fueron emplazadas en contextos de dunas y antiguas terrazas marinas y relevado 2 veces en el lapso de 2 años, y en el caso 2 las pistas experimentales se emplazaron sobre sendas animales activos y el experimento se relevó 3 veces en el lapso de 4 años. Más detalle sobre la realización de los experimentos ha sido previamente publicado (Ver Balirán, 2014; Carranza, 2017).

\section{Caso de estudio 1: ambientes eólicos en el golfo San Matías}
Área de estudio
El sector norte del golfo San Matías se extiende desde la ciudad de San Antonio Oeste hasta el Balneario El Cóndor, en la desembocadura del río Negro en el océano Atlántico (Río Negro,

Figura 1:. Mapa donde se señalan las tres áreas bajo estudio. A: golfo San Matías, Provincia de Río Negro; B: sur de sierra Baguales, provincia de Santa Cruz; C: microrregión de Antofagasta de la Sierra, provincia de Catamarca.

Figure 1. Map showing the three areas under study. A: San Matías gulf, Río Negro province; B: southern sierra Baguales, Santa Cruz province; C: Antofagasta de la Sierra microregion, Catamarca Province. 
Argentina) (Figura 1). Allí se han recuperado conjuntos líticos de superficie en diferentes loci en contextos de dunas y terrazas (Carranza, 2017). Esta costa se caracteriza por un clima semi-árido con precipitaciones de $250 \mathrm{~mm}$ anuales y con vientos máximos de $123 \mathrm{~km} / \mathrm{h}$. La vegetación es de tipo monte oriental, con poca cobertura (Oyarzabal et al., 2018). En la costa norte del golfo las fuentes de materias primas líticas son principalmente secundarias, con rocas de diferentes orígenes (volcánicas, sedimentarias, piroclásticas y metamórficas) (Favier Dubois y Alberti, 2014). Los mantos eólicos y campos de dunas se acumulan en toda la costa sobre antiguas terrazas marinas, siendo tanto de carácter móvil como en forma de dunas fijas por la vegetación (Favier Dubois, Kokot, Scartascini y Borella, 2016). La ocupación humana de la costa data desde al menos 6.000 años AP, hecho que está sostenido por evidencia artefactual, faunística y bioarqueológica (Borella y Cardillo, 2011). Dado que las evidencias arqueológicas se depositan en ambientes predominantemente eólicos, es esperable que los loci hayan estado en superficie o enterrados en distintas oportunidades y que existan sectores actualmente destruidos, sepultados o en los que los materiales se encuentren redepositados por acción del viento (Favier Dubois et al., 2016).

\section{Evaluación del problema}

El objetivo del trabajo fue evaluar en qué medida la estructuración y distribución diferencial de los conjuntos líticos en el área de estudio eran producto de los procesos de formación del registro. Para ello se analizaron las alteraciones registradas en los artefactos líticos como indicadores del grado de acción de los agentes tafonómicos.

\section{Resultados obtenidos y} discusión

En términos generales, los estadios de corrasión mayormente registrados son el 1 y 2, es decir, corrasión leve a avanzada. Los loci ubicados en geoforma de terrazas marinas poseen mayor intensidad de corrasión, mientras que los loci ubicados en contextos de dunas registran estadios de corrasión menores (mayoría piezas sin alteraciones sobre su superficie) (Carranza, 2017). La presencia de carbonatación se registró en todos los loci en bajas proporciones. La disponibilidad de sedimentos movilizados por el viento "limpia" las superficies de los artefactos impidiendo su formación. Por eso, el registro de carbonatos en este ambiente sugeriría una exposición reciente de los conjuntos. Los resultados obtenidos en el análisis tafonómico de las muestras arqueológicas mostraron que los tempos de destape de los conjuntos varían en las diferentes localizaciones en correspondencia con los ciclos erosivos. La evaluación de estabilidad sugiere la existencia de variabilidad de condiciones dependiendo de los contextos geomorfológicos de depositación (Carranza, 2017). Los conjuntos emplazados en terrazas marinas registran condiciones más estables, mientras que los conjuntos en loci localizados en sectores de dunas registran condiciones menos estables. El análisis de la granulometría artefactual informó sobre la formación de concentraciones re-depositadas por acción del viento (piezas con un volumen mínimo de 0.02 a 1.26 de máximo) (Figura 2). En cuanto a los datos experimentales, el primer relevamiento realizado 19 meses después del sembrado de las pistas mostró cambios significativos en todas las variables registradas (desplazamientos horizontales de hasta $145 \mathrm{~cm}$.; inversión; ver Carranza, 2017). La experimentación in situ permitió conocer la magnitud mínima de los cambios que el viento puede provocar y aportó los primeros valores de referencia locales. En este sentido, confirmamos que la remoción de piezas por acción del viento tiene lugar y que ella tiene el potencial de sesgar la interpretación

Figura: 2. A: formación de concentraciones re-depositadas por acción del viento; B: filos con grados de meteorización menores ("frescos"); C: talleres líticos de variada extensión y densidad con desechos de talla y materiales descartados.

Figure 2. A: lithic concentration by wind action; B: edges with low weathering ('fresh'); C: quarry workshops of varied extension and density with knapping debris and discarded materials. 
que se realiza de los conjuntos artefactuales (Borrazzo, 2006, 2016). La combinación de resultados obtenidos, sumado a la información distribucional del área, sugiere que la interacción entre procesos eólicos (corrasión, transporte y depositación) y las características del conjunto (composición granulométrica) influyen sobre las características de los conjuntos arqueológicos que hallamos actualmente.

\section{Caso de estudio 2: localidad arqueológica La Verdadera Argentina}

\section{Área de estudio}

La localidad arqueológica La Verdadera Argentina (LVA) se encuentra ubicada al sudeste de sierra Baguales (Santa Cruz, Argentina) (Borrero et al., 2006). Emplazada entre 300 y 600 m.s.n.m., registra precipitaciones anuales de $300 \mathrm{~mm}$, una temperatura media anual de $8^{\circ} \mathrm{C}$ y vientos de hasta $120 \mathrm{~km} / \mathrm{h}$. La vegetación es de tipo estepa arbustiva baja (Oyarzabal et al., 2018) y la fauna cuenta tanto con especies silvestres, entre ellas guanaco (Lama guanicoe), zorro gris (Lycalopexgriseus), choique (Rheapennata) y puma (Puma concolor); como ganado (caballo, oveja y vaca) (Figura 1). Los recursos líticos, disponibles principalmente en fuentes de tipo secundario, se presentan en forma de rodados y/o grandes bloques poco redondeados que evidencian escaso transporte.

Las materias primas incluyen ftanita, dacita, diabasa y rocas lutíticas. Estas últimas dominan los conjuntos artefactuales de LVA (Borrazzo, 2008). La localidad cuenta con cronologías para las ocupaciones correspondientes a todo el Holoceno, con fechados entre 8.856 \pm 84 y $907 \pm 45$ años ${ }^{14} \mathrm{C}$ AP (Borrero et al., 2006; Borrazzo, 2008; Borrero y Borrazzo, 2011; Balirán, 2014). A pesar de esta larga cronología, LVA se ha caracterizado por una señal arqueológica poco intensa manifestada en la ausencia de evidencias de uso humano en espacios que ofrecen condiciones adecuadas para su utilización (e.g. cueva LVA, Borrazzo, 2008). Adicionalmente ha sido considerada como un área marginal dentro de los rangos de acción de las poblaciones localizadas al Este de este espacio (Franco y Borrero, 2000; Borrero et al., 2006; Borrazzo, 2008). En este contexto general se ha observado que el registro lítico de superficie de LVA, en particular aquellos artefactos confeccionados sobre rocas lutíticas, presentan diversos grados de meteorización química en sus superficies talladas. En estos casos son siempre los filos los que presentan los menores grados de meteorización (i.e. están más "frescos") (Figura 2). Se plantearon dos hipótesis alternativas para explicar dicho patrón: $H_{0}$ - el patrón observado es producto antrópico intencional, específicamente causado por el proceso de reclamación (sensu Schiffer, 1987); $H_{1}$. el patrón observado es resultado de causas tafonómicas, específicamente producto del pisoteo de animales.

Evaluación del problema
El objetivo entonces fue detectar, a partir del estudio de variables tafonómicas (Ver Metodología), la incidencia de diversos procesos que pudieron actuar o que actúan sobre los conjuntos líticos de superficie hallados en LVA y la intensidad con la que pudieron afectar a los mismos. En relación con las hipótesis planteadas, mientras que la presencia de animales de porte adecuado en el área hacía factible la $H_{1}$, las cronologías para las ocupaciones humanas de LVA correspondientes a distintos momentos del Holoceno hacía factible también la $H_{0}$. Con el fin de evaluar la incidencia del pisoteo animal se decidió realizar un programa experimental. Como se mencionó más arriba, el experimento incluyó el sembrado de pistas experimentales con artefactos confeccionados sobre lutita $(\mathrm{N}=100)$. Las variables registradas durante el sembrado de las pistas y sus posteriores relevamientos fueron: cambio en la cara expuesta de las piezas y estado de estas (i.e. enteras/fracturadas o 'lascadas') (Balirán, 2014).

\section{Resultados obtenidos y discusión}

El análisis tafonómico de la muestra artefactual $(\mathrm{N}=307)$ mostró que el $91 \%$ de los artefactos $(\mathrm{N}=282)$ presentaba meteorización (estadio 0: 8,1\%; estadio 1: 41,4\%; estadio 2: 41\%; estadio 3: 9,1\%). De esos artefactos, 152 casos presentaron más de un estadio de meteorización sobre su superficie, de los cuales en 141 artefactos el menor estadio de meteorización correspondía con los lascados observados sobre los filos. Sin embargo, los filos exhibieron sólo los estadios $1(\mathrm{~N}=7)$ y $0(\mathrm{~N}=134)$. Ello muestra una variabilidad menor que la reflejada por los estadios máximos de meteorización. Este fenómeno -que informa sobre una cierta sincronía relativa de las modificaciones que exhiben el mismo estadio de meteorización- puede deberse a que los artefactos que exhiben esta característica tienen una historia formacional relativamente similar, que estas superficies han quedado expuestas recientemente y durante un lapso no muy prolongado de tiempo.

En cuanto al experimento, se pudieron observar cambios significativos en las variables registradas. En los relevamientos llevados a cabo, se pudo observar que hubo cambios en la cara expuesta de las piezas (32\% en 2012 , $26 \%$ en 2014 y $18 \%$ en 2015 ). Si bien esta variable registra el número mínimo de inversiones su importancia reside en que da cuenta de movimientos producto del pateo y pisoteo de distintos animales (Somonte et al., 2004). Asimismo, a lo largo de los tres relevamientos un $36 \%$ de piezas presentó microlascados de origen tafonómico sobre los bordes de las piezas, en los que se contaron un total de 112 bocas de lascado. En cuanto a las fracturas, en los 4 años relevados se observó un 22\% de piezas fracturadas con un total de 34 fracturas. Esto ha sido útil como dato proxy para evaluar la intensidad del pisoteo animal. Estos resultados experimentales dan cuenta de la dinámica a la que se encuentra expuesto el registro lítico de superficie y serán útiles, como marco de referencia, para revisar los datos obtenidos en los análisis 
tafonómicos y tecnomorfológicos realizados sobre otras muestras de LVA. Asimismo, si bien no son suficientes para descartar la hipótesis antrópica $\left(H_{1}\right)$, refuerzan la factibilidad de la hipótesis tafonómica $\left(H_{0}\right)$. Por lo tanto, los resultados obtenidos no solo ponen de manifiesto la presencia y potenciales efectos de los procesos tafonómicos sobre el registro lítico de superficie en LVA, sino también informan sobre su intensidad. Finalmente se destaca que los resultados experimentales han sido útiles para generar modelos aplicables a casos que presenten una problemática similar a pesar de encontrarse en contextos que difieran del aquí planteado (Weitzel, Borrazzo, Ceraso y Balirán, 2014).

\section{Caso de estudio 3: canteras-taller de la microrregión de Antofagasta de la Sierra}

\section{Área de estudio}

La microrregión de Antofagasta de la Sierra está emplazada a 3323 m.s.n.m., es una región semiárida y/o desértica con $124 \mathrm{~mm}$ de precipitaciones anuales y marcada estacionalidad (Figura 1). Su vegetación es dispersa y xerófila (Oyarzabal et al., 2018). Los vientos alcanzan un promedio de $24,89 \mathrm{~km} / \mathrm{h}$ y una máxima de $60,83 \mathrm{~km} / \mathrm{h}$ que generan tormentas de arena y polvo (Tchilinguirian, 2008). Particularmente, en el área de canteras-taller, el desarrollo de suelo es pobre, con baja potencia estratigráfica. En superficie se distribuyen clastos y bloques de volcanitas que presentan facetas y ventifacción (García Salemi, 1986; Tchilinguirian, 2008). En estas canteras-taller, se llevaron a cabo actividades de aprovisionamiento durante una extensa secuencia de ocupación (desde los 10.000 años AP, aproximadamente) (Pintar, 1996; Toselli, 1998; Aschero et al., 20022004; Manzi, 2006; Bobillo y Hocsman, 2015; entre otros). El producto de estas actividades (desechos de talla y materiales descartados) quedó depositado en superficie, formando talleres líticos de variada extensión y densidad (Figura 2). Así, al tratarse de áreas con poca vegetación, escasa potencia sedimentaria, condiciones geomorfológicas variables y alta exposición eólica se requiere de una evaluación de los procesos de formación. Dicha evaluación es un paso importante como parte del programa de investigación arqueológica de las canterastaller.

\section{Evaluación del problema}

El objetivo fue estudiar el grado de preservación y la integridad de los conjuntos líticos superficiales que se distribuyen en las canteras-taller. Para ello se analizaron las configuraciones espaciales que presentan los conjuntos líticos en superficie, ya que se reconocieron diferentes niveles de densidad y agrupamiento de materiales tallados y en estado natural; como así también conjuntos que yacían sobre geoformas variables. Además, como parte del proceso de evaluación de la integridad del registro arqueológico se llevó a cabo un estudio de granulometría lítica (Bertran et al., 2012; Lenoble y Bertran, 2004) en tres talleres a cielo abierto del área de canteras-taller. Por otra parte, con el fin de reconocer eventos de talla diacrónicos y estimar cronología relativas -dada la escasez de materiales que posibilitaran una datación absolutase analizó la intensidad de la abrasión eólica superficial (corrasión) de distintas clases tipológicas (Borrazzo, 2010, 2016).

\section{Resultados alcanzados y discusión}

A partir de las observaciones en el campo se pudieron reconocer condiciones variables de preservación de los conjuntos líticos y su relación con los procesos postdepositacionales que actúan en el área de canterastaller. Es así como se distinguieron talleres extensos con elevada densidad de materiales tallados (entre 30 y 70 $\mathrm{m}$ de diámetro) y eventos de talla acotados en el espacio (entre 4 y $10 \mathrm{~m}$ de diámetro). Estos últimos poseen menor densidad de materiales y límites bien definidos. En ambos tipos de talleres (extensos o acotados/discretos) las partículas líticas presentan un nivel de agrupamiento elevado. Por otra parte, se registraron sectores donde los elementos poseen un bajo nivel de agregación y se encuentran dispersos sobre la superficie de las canterastaller. En relación con la presencia/ausencia de la fracción más pequeña, se observó que los elementos que poseían tamaños inferiores al centímetro son los que menor frecuencia tienen en los tres talleres a cielo abierto. La baja frecuencia (15\%) que posee esta fracción sería el resultado de la incidencia de agentes erosivos como el viento (Borrazzo, 2016). Con respecto al grado de agrupamiento y dispersión de las piezas, se reconocieron talleres con baja incidencia de procesos postdepositacionales, localizados en áreas planas o ligeramente inclinadas; estos talleres muestran un elevado grado de agregación entre partículas. Por otro lado, se reconocieron geoformas donde las partículas se encuentran desagregadas entre sí, y en donde las relaciones inter-espaciales de los elementos se perdieron. Factores erosivos como el viento o la escorrentía superficial habrían influido en la desagregación de dichos componentes. Asimismo, en algunas geoformas se observaron artefactos y lascas que presentan un "acomodamiento" lineal que se circunscribe a lo que podrían ser canales de origen pluvial. Esto sería el resultado de la caída y circulación de agua durante precipitaciones estivales. La aplicación de técnicas de estudio como la granulometría lítica, remontaje y fábrica (Cziesla, 1990; Bertran et al., 2012; Lenoble y Bertran, 2004) -actualmente en vía de desarrollo- aportarán información para comprender el grado de preservación o transformación de los contextos líticos superficiales.

En lo referido al estudio de las intensidades diferenciales de abrasión eólica, se reconocieron distintos estadios en los materiales líticos tallados. Esto permitió estimar una cronología relativa de las actividades de talla. Al respecto, debe considerarse que los datos reunidos se encuentran aún en vía de estudio, ya que para poder asociar completamente estadios de abrasión eólica con un 
componente cronológico se requiere de una evaluación de distintos proxies vinculados con las características del entorno de los depósitos (Ugalde et al., 2015). Dado que estos múltiples proxies se encuentran aún en proceso de análisis, los estadios diferenciales de abrasión se consideraron en función de las asociaciones que presentan con artefactos tecno-tipológica y temporalmente diagnósticos datados en sitios residenciales con estratigrafía. A partir de esta información se pudieron reconocer momentos de una secuencia temporal en la que se habrían explotado las canteras-taller (para más detalle de estas asociaciones véase Bobillo y Hocsman, 2015). Por otra parte, la identificación de estadios diferenciales de abrasión eólica permitió reconocer eventos de talla diacrónicos (Bobillo, 2014). Dichos eventos se definieron a partir de la identificación de piezas que poseen superposición de planos con estadios de abrasión distintos. Particularmente, se reconocieron núcleos que presentan superficies con estadios de abrasión más severos sobre las que se superponen otros planos de lascado con estadios menos intensos, como así también lascas y artefactos formatizados que tienen superficies superpuestas con abrasión diferencial. Todo ello, indicaría la reutilización de piezas en distintos momentos de uso y explotación de las canteras-taller.

\section{Discusión y Conclusiones}

Los contextos líticos superficiales aquí abordados se encuentran sujetos al accionar de distintos agentes, en este caso, principalmente, el viento y los animales. Los efectos relevados incluyeron transformaciones de índole cuantitativa y cualitativa a nivel del artefacto y de los conjuntos. Si bien en los tres casos el ambiente es similar, a partir de la comparación, destacamos que las observaciones naturalistas han sido de gran utilidad para calibrar cada caso a su problemática local (Marean, 1995). Mientras en el caso 1 y 3 se detectó que el principal agente tafonómico era el viento, combinado con la disponibilidad de sedimentos (i.e. arena y arcillas), en el caso 2 fue el pisoteo de animales. Si bien otros agentes no están ausentes, en los tres casos se consideró que su efecto no era suficiente como para alterar las interpretaciones realizadas a partir del estudio de los conjuntos. Definir el agente tafonómico permitió seleccionar las variables adecuadas para evaluar cada caso. En concordancia con ello los casos 1 y 3 seleccionaron la corrasión para medir los efectos del transporte eólico de los sedimentos, esto posibilitó evaluar alteraciones morfológicas de las piezas que permitieron diacronía en la exposición subaérea de los conjuntos. Algo similar se observó en el caso 2, en el que el análisis de la meteorización química de los artefactos dió cuenta de la diacronía entre su confección y la de algunos de sus filos. A partir de ello se planteó como hipótesis que ese fenómeno podría ser producto del pisoteo animal. Por otro lado, en los casos 1 y 3, a través del análisis de granulometría se constató la ausencia de las partículas más pequeñas en los conjuntos líticos. Esto sugiere el accionar del viento y escorrentía superficial como los principales agentes de transformación. El estudio tafonómico de cada caso permitió establecer hipótesis acerca de la influencia del ambiente en los conjuntos estudiados. En este sentido en los casos 1 y 2 se decidió que una vía clave para evaluar las hipótesis tafonómicas era la experimentación. En ambos casos se decidió realizar experimentos de seguimiento longitudinal siguiendo la propuesta de Borrero (1991). Se destaca en ambos casos que los resultados experimentales no sólo son de gran importancia para comprender y calibrar a nivel local la incidencia de los procesos tafonómicos que afectan los conjuntos estudiados, sino que también sus resultados pueden ser utilizados para comprender otros conjuntos disponibles a nivel regional que presenten características similares. En el caso 3, si bien no incluyó la experimentación, la misma es considerada para ser aplicada en trabajos actualmente en desarrollo.

De los casos presentados se desprenden algunas conclusiones que resultan interesantes de destacar acerca de la aplicación del enfoque tafonómico. En primer lugar, se destaca la importancia de las observaciones naturalistas, que estén orientadas a conocer la dinámica local para comprender sus efectos sobre el registro arqueológico. En este sentido, incluirlas dentro de un enfoque geoarqueológico permite estudiar el modelado del registro por factores ambientales. En función de esto una cuestión que resultó de la comparación de los tres casos, es que, a pesar de tratarse de registros ubicados en contextos ambientales similares, es necesario calibrar y entender los problemas en una escala local. La importancia de esto reside en que los agentes identificados pueden variar de un espacio a otro $u$ afectar de forma diferente al registro. En este sentido las variables analizadas (e.g. el emplazamiento en el terreno, desplazamiento horizontal y vertical de las piezas, el grado de agregación de partículas líticas, fractura de artefactos, entre otros) son de suma importancia al momento de evaluar el grado de transformación que tienen los contextos superficiales con los cuales se trabaja. Finalmente, estos tres casos dan cuenta de la utilidad de implementar un enfoque tafonómico como así también la experimentación como vía de testeo de hipótesis. Ambos permiten mejorar nuestra comprensión de los procesos postdepositacionales y ajustar nuestras interpretaciones del pasado, evitando sobreestimar la dimensión de la agencia humana en la variabilidad observada.

Ciudad Autónoma de Buenos Aires, 15 de abril 2019

\section{Agradecimientos.}

A los organizadores del $1^{\circ}$ Congreso Argentino de Estudios Líticos en Arqueología. A CONICET por el financiamiento otorgado. A los proyectos: PIUNT G503, PIP-CONICET 577, PICT 1703, UBACyT 046, UBACyT 20020100100957, PIP 11220110100262. A la Dra. Karen Borrazzo por sus 
enriquecedores comentarios. A los evaluadores anónimos por sus valiosos comentarios y sugerencias que sin duda mejoraron el trabajo sustancialmente.

\section{Referencias citadas.}

Aschero, C. A., Hocsman, S., y Martínez, J. G. (2002-2004). Recursos líticos en escala microrregional, Antofagasta de la Sierra, 1983-2001. Arqueología, (12), 9-36.

Balirán, C. (2014). Trampling, taphonomy, and experiments with lithic artifacts in the southeastern Baguales Range (Santa Cruz, Argentina). Intersecciones en antropología, 15(1), 85-95.

Bertran, P., Lenoble, A., Todisco, D., Desrosiers, M. P. y Sørensen, M. (2012). Particle size distribution of lithic assemblages and taphonomy of Palaeolithic sites. Journal of Archaeological Science, 39, 3148-3166.

Bobillo, F. M. (2014). Actividades y estrategias tecnológicas en canteras de vulcanita en la localidad arqueológica de Punta de la Peña (Antofagasta de la Sierra - Catamarca) (Tesis de grado inédita). Facultad de Ciencias Naturales e Instituto Miguel Lillo. Universidad Nacional de Tucumán. Argentina.

Bobillo, F. M., y Hocsman, S. (2015). Mucho más que solo aprovisionamiento lítico: actividades en canteras y prácticas sociales en las fuentes de Pampa Oeste, Quebrada Seca y Punta de la Peña (Antofagasta de la Sierra, Catamarca). Revista Museo de Antropología, 8(1), 23-44.

Borella, F., y Cardillo, M. (2011). Arqueología de Pescadores y Marisqueadores en Nordpatagonia. Buenos Aires. Editorial Dunken.

Borrazo, K. (2006) Tafonomía lítica en dunas: una propuesta para el análisis de los artefactos líticos. Intersecciones en Antropología, 7, 247-261.

Borrazo, K. (2008). Análisistecnológico de distribucionesartefactuales en la periferiasudeste de la sierra Baguales (Santa Cruz, Argentina). Magallania (Punta Arenas), 36(1), 103-116.

Borrazo, K.(2010). Arqueología de los estepariosfueguinos. Tafonomía y tecnologíalítica en el norte de Tierra del Fuego, Argentina (Tesis doctoral inédita). Facultad de Filosofía y Letras. Universidad de Buenos Aires, Buenos Aires.

Borrazo, K. (2016). Lithic taphonomy in desert environments: Contributions from Fuego-Patagonia (Southern South America). Quaternary International, 422, 19-28.
Borrero, L. A. (1991). Experimentos y escalas arqueológicas. Shincal, 3(1), 142.

Borrero, L. A. (2001). Regional taphonomy: background noise and the integrity of the archaeological record. Ethnoarchaeology of Andean South America. Contributions to archaeological method and theory, 243-254.

Borrero, L. A., y Borrazzo, K. (2011). La geografía cultural del sudoeste de Patagonia continental. Bosques, montañas y cazadores. Investigaciones Arqueológicas en Patagonia Meridional, 7-36.

Borrero, L. A., Franco, N. V., Martin, F., Barberena, R., Guichón, R., Belardi, J. B., Favier Dubois, C. y L'Heureux, L. (2006). Las cabeceras del Coyle: información arqueológica y circulación de poblaciones humanas. En J.B. Belardi, F. Carballo Marina y S. Espinosa (Eds.), La cuenca del río Coyle (pp. 75-95). Río Gallegos: UNPA.

Butzer, K. (1982). Arqueología, una ecología del hombre: método y teoría para un enfoque contextual. Cambridge: University Press.

Carranza, E. (2017). Análisis tafonómico de conjuntos líticos de superficie en la costa norte del golfo San Matías (Rio Negro, Argentina). Intersecciones en Antropología, 18(1), 91-101.

Cziesla, E. (1990). On refitting of stone artefacts. The Big Puzzle: International Symposium on Refitting Stone Artefacts, Studies in Modern Archaeology Vol. 1, pp. 9-44.

Domínguez-Rodrigo, M., Fernández-López, S., y Alcalá, L. (2011). How can taphonomy be defined in the XXI Century. Journal of Taphonomy, 9 (1), 1-13.

Dorn, R. I. (2009). Desert rock coatings. En A.J. Parsons, A.D. Abrahams (Eds.), Geomorphology of desert environments (pp. 153-186). Dordrecht: Springer.

Favier Dubois, C., y Alberti, J. (2014). Materias primas líticas en la costa norte del Golfo San Matías (Río Negro, Argentina): Distribución de fuentes y tendencias generales en su aprovechamiento. Revista del Museo de Antropología, 7(1), 93-104.

Favier Dubois, C., Kokot, R., Scartascini, F., y Borella, F. (2016). Una perspectiva geoarqueológica del registro de ocupaciones humanas en el Golfo San Matías (Río Negro, Argentina). Intersecciones en Antropología, 17 (1083), 47-59.

Franco, N. V., y Borrero, L. A. (2000). Estrategias de utilización de Sierra Baguales. Contribución Arqueológica, 5, 269-283. 
García Salemi, M. (1986). Geomorfología de regiones secas: Antofagasta de la Sierra, Provincia de Catamarca. Centro de Estudios Regiones Secas, 4(1-2), 5-13.

Hiscock, P. (1985). The need for a taphonomic perspective in stone artefact analysis. Queensland Archaeological Research, 2, 82-97.

Lenoble, A., y Bertran, P. (2004). Fabric of Palaeolithic levels: Methods and implications for site formation processes. Journal of Archaeological Science, 31(4), 457-469.

Lyman, R. L. (1994). Vertebrate taphonomy. Cambridge University Press.

Manzi, L. M. (2006). Estrategias y formas de uso del espacio en poblaciones cazadoras recolectoras de la Puna meridional argentina. Archaeopress.

Marean, C. (1995). Of Taphonomy and Zooarchaeology. Evolutionary Anthropology, 4(2), 64-72.

Oyarzabal, M., Clavijo, J., Oakley, L., Biganzoli, F., Tognetti, P., Barberis, I., Maturo, H.M., Aragón, R., Campanello, P.I., Prado, D., Oesterheld, M. y León, R.J.C. (2018). Unidades de vegetación de la Argentina. Ecología Austral 28,40-63.

Pintar, E. (1996). Prehistoric Holocene Adaptations to the Salt Puna of Northwestern Argentina. (Tesis doctoral inédita). Graduate Faculty of Dedman College, Southern Methodist University, Texas.

Schiffer, M. B. (1972). Society for American Archaeology
Archaeological Context and Systemic Context. American Antiquity, 37 (2), 156-165.

Schiffer, M. B. (1987). Formation processes of the archaeological record. Albuquerque: University of New Mexico Press.

Somonte, C., Hocsman, S., Martel, A. R., y Babot, M. P. (2004). Procesos de formación en un sitio cantera-taller: Campo Blanco (Tucumán, Argentina). Chungara Revista de Antropología Chilena, 36, 983-995.

Tchilinguirian, P. (2008). Paleoambientesholocénicos en la puna austral, provincial de Catamarca $\left(27^{\circ} \mathrm{S}\right)$ : Implicancias geoarqueológicas (Tesis doctoral inédita), Universidad Nacional de Buenos Aires, Argentina.

Toselli, A. (1998). Selección de materias primas líticas y organización tecnológica en el sitio Punta de la Peña 4 (PP4), Depto. Antofagasta de la Sierra, Prov. de Catamarca (Tesis de grado inédita). Facultad de Ciencias Naturales e Instituto Miguel Lillo. Universidad Nacional de Tucumán, Argentina.

Ugalde, P. C., Santoro, C. M., Gayo, E. M., Latorre, C., Maldonado, S., Pol-Holz, R. D. y Jackson, D. (2015). How do surficial lithic assemblages' weather in arid environments? A case study from the Atacama Desert of northern Chile. Geoarchaeology 30, 352 - 368.

Weitzel, C., Borrazzo, K., Ceraso, A., y Balirán, C. (2014). Trampling Fragmentation Potential of lithic artifacts: an experimental approach. Intersecciones en Antropología, 15(1), 97-110. 